\title{
P49 Experimental Flow Visualization of a Flow Diverting Stent in a Popliteal Aneurysm
}

\author{
Lennart van de Velde ${ }^{1,2,3, *}$, Michel Reijnen ${ }^{4,5}$, Michel Versluis ${ }^{6}$, Joerg Tessarek ${ }^{7}$, Erik Groot Jebbink ${ }^{5,4}$ \\ ${ }^{1}$ University of Twente, Enschede, The Netherlands \\ ${ }^{2}$ Amsterdam UMC, Location AMC, Amsterdam, The Netherlands \\ ${ }^{3}$ Rijnstate Hospital, Arnhem, The Netherlands \\ ${ }^{4}$ Department of Surgery, Rijnstate Hospital, Arnhem, The Netherlands \\ ${ }^{5}$ M3i Multi-Modality Medical Imaging, TechMed Centre, University of Twente, Enschede, The Netherlands \\ ${ }^{6}$ Physics of Fluids, TechMed Centre, University of Twente, Enschede, The Netherlands \\ ${ }^{7}$ Department of Vascular Surgery, Bonifatius Hospital, Lingen, Germany
}

\section{ABSTRACT}

Purpose: Flow diverting stents are commonly used in saccular cerebral aneurysms [1]. Their use has not yet been investigated for popliteal aneurysms, which are commonly fusiform in nature and exposed to pulsatile triphasic flows. We have shown the clinical feasibility of flow diverters in a pilot study of 12 patients [2]. To investigate its physiologic mechanism and the optimal flow diverter configuration, flow profiles were investigated in popliteal aneurysm phantoms.

Methods: Six transparent silicone elastomer models were created and integrated in an experimental flow set-up, which reproduced physiologic pulsatile flow conditions. These models covered two idealized anatomies (Figure 1) and three stent configurations (control, single stent [SUPERA, Abbott, Santa Clara, CA] and dual stent). Laser particle image velocimetry was used to investigate two-dimensional flow velocities, shear rates and wall shear stress in the models.

Results: The introduction of a flow diverter stent led to a two-time reduction in aneurysm flow velocities for single-layer stents, and a three-time reduction for double layer stents (Figure 2), with higher reduction factors for the bent inlet model. Forward flow was optimally diverted, whereas backward flow was deflected into the aneurysm by the unceasing momentum of the forward jet. Nonetheless, both flow velocities, shear rates (1.3-2x decrease) and wall shear stress (2.8-4.5x decrease) were strongly reduced by the double-layer configuration.

Conclusion: This study demonstrates the ability of a double-layer flow diverter stent for reducing flow velocities in popliteal aneurysms, supporting its clinical use in terms of its fluid mechanical effects.
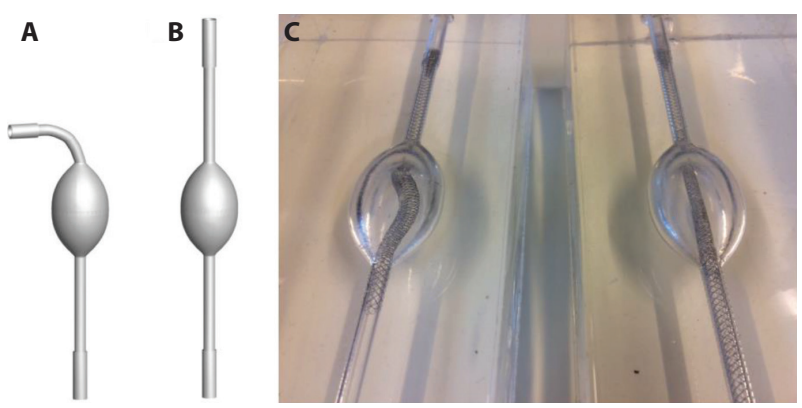

Figure 1 The popliteal aneurysm phantoms. (A) Bent inlet trajectory. (B) Straight inlet trajectory. (C) The straight inlet model treated with a single layer stent (left) and a dual layer stent (right), which follow a helical and straight trajectory, respectively. 

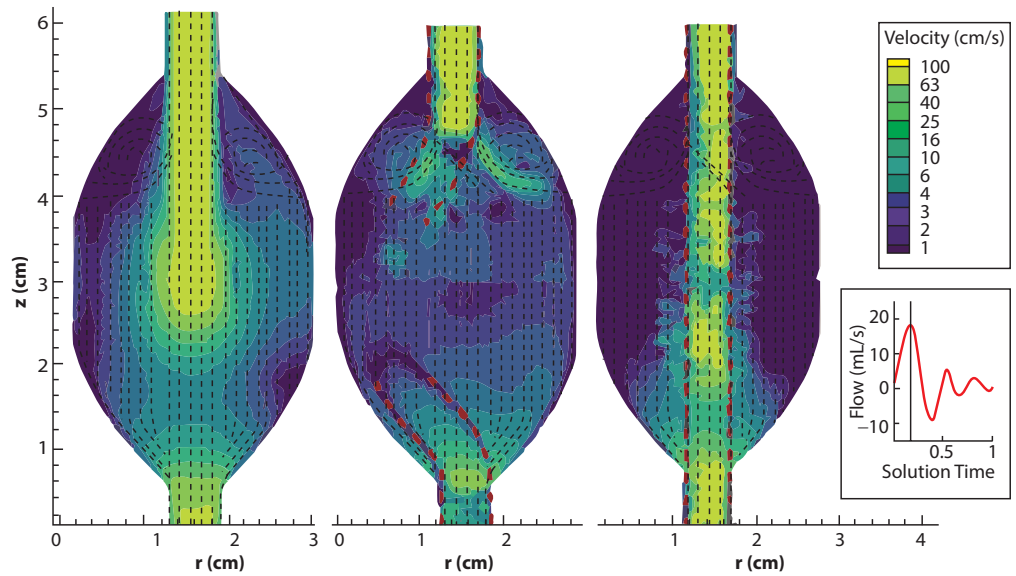

Figure 2 Velocity vector and contour plots during peak forward flow in the straight inlet models. From left to right: control, single layer (dashed red line) and double layer (dash-dot red line) stent. The single layer stent is partly out-ofplane due to its helical trajectory.

\section{REFERENCES}

[1] Alderazi YJ, Shastri D, Kass-Hout T, Prestigiacomo CJ, Gandhi CD. Flow diverters for intracranial aneurysms. Stroke Research and Treatment 2014;2014:415653.

[2] Tessarek J. Zentralblatt Chirurgie 2015;140:535-41.

(C) 2019 Association for Research into Arterial Structure and Physiology. Publishing services by Atlantis Press International B.V. This is an open access article distributed under the CC BY-NC 4.0 license (http://creativecommons.org/licenses/by-nc/4.0/). 